Research Paper

\title{
Lymphocyte/Monocyte Ratio is a Novel Predictor for Early Stage Extranodal Natural Killer/T-cell Lymphoma, Nasal Type
}

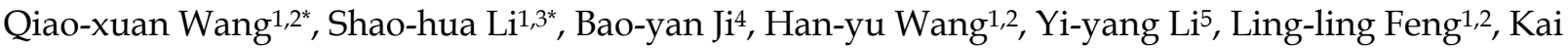 \\ Chen ${ }^{1,2 \bowtie}$, Yun-fei Xia ${ }^{1,2}$, Yu-jing Zhang ${ }^{1,2}$ \\ 1. State Key Laboratory of Oncology in South China; Collaborative Innovation Center for Cancer Medicine, Guangzhou, P R. China; \\ 2. Department of Radiation Oncology, Sun Yat-sen University Cancer Center, Guangzhou, P. R. China; \\ 3. Department of Hepatobiliary Oncology, Sun Yat-sen University Cancer Center, Guangzhou, P. R. China; \\ 4. Department of Oncology, Qinghai Province People's Hospital; \\ 5. Department of Oncology, the First Affiliated Hospital of Guangdong Pharmaceutical University. \\ * These authors contributed equally to this work. \\ $\triangle$ Corresponding authors: Yu-jing Zhang, M.D., Tel: 0086-020-87343702; Fax: 0086-020-87343392; E-mail: yujing_zhang1969@163.com. Kai Chen, M.D., Tel: \\ 0086-020-87343702; Fax: 0086-020-87343392; E-mail: chenkai@sysucc.org.cn. \\ (c) Ivyspring International Publisher. This is an open access article distributed under the terms of the Creative Commons Attribution (CC BY-NC) license \\ (https:// creativecommons.org/licenses/by-nc/4.0/). See http://ivyspring.com/terms for full terms and conditions.
}

Received: 2016.08.30; Accepted: 2016.12.16; Published: 2017.04.08

\begin{abstract}
Objective: Great heterogeneity exists in clinical behavior and survival outcome in patients with stage IE/IIE extranodal natural killer/T-cell lymphoma, nasal type (ENKTL). In this study, we proposed lymphocyte/monocyte ratio (LMR) as a new prognostic factor for these early stage ENKTL.

Methods: We retrospectively examined the LMR as a prognostic variable in a cohort of 379 patients with newly diagnosed stage IE/IIE ENKTL. The relationship between the LMR and clinicopathologic variables were analyzed in Kaplan-Meier log-rank survival analysis, and the Cox proportional hazards model was used to determine the survival significance of the LMR for both progression-free survival (PFS) and overall survival (OS).

Results: Patients were categorized into two different groups based on the LMR using cut-off value of 2.0. The 5-year PFS rates in the low and high LMR group were $43.9 \%$ and $62.7 \%$, respectively, and the 5 -year OS rates in the two groups were $59.1 \%$ and $77.7 \%$, respectively. In multivariate analysis, low LMR at diagnosis was associated with worse PFS (hazard ratio $1.611,95 \%$ confidence interval: 1.027-2.525, $\mathrm{P}=0.038)$ independent of age $(\mathrm{P}=0.033)$ and treatment stratagem $(\mathrm{P}<0.001)$, and indicated worse OS (hazard ratio $2.003,95 \%$ confidence interval: 1.124-3.569, $\mathrm{P}=0.018$ ) independent of age $(P=0.007)$, LDH level $(P=0.042)$, local tumor invasiveness $(P=0.008)$, and treatment stratagem $(P<0.001)$.

Conclusion: The LMR is an independent prognostic factor for both DFS and OS in patients with stage IE/IIE ENKTL, and provides additional prognostic value beyond standard clinicopathological parameters.
\end{abstract}

Key words: extranodal natural killer/T-cell lymphoma, lymphocyte/monocyte ratio, prognosis

\section{Introduction}

Extranodal natural killer/T-cell lymphoma, nasal type (ENKTL), is a distinct histopathologic subtype of non-Hodgkin's lymphoma according to the new World Health Organization (WHO)
Classification, characterized by vascular damage and destruction, obvious necrosis, cytotoxic phenotype, and related to Epstein-Barr virus (EBV)[1]. Although it is quite rare in western populations, ENKTL is more 
prevalent in East Asia and makes up approximately $5-10 \%$ of all lymphomas in the Chinese population[2].

Patients who presented with ENKTL are staging using the Ann Arbor staging system for non-Hodgkin's lymphoma. Stage IIIE/IVE ENKTL exhibited more aggressive tumor behavior and poorer prognosis compared with patients of stage IE/IIE. However, there were quite a lot of cases with stage I/II ENKTL who had poor prognosis because of rapid disease progression and recurrence, and the Ann Arbor stage failed to predict survival differences between stage IE and stage IIE in some studies[3]. The International Prognostic Index (IPI)[4] was also used for assessing the prognosis of patients with ENKTL. Though validated in many subtypes of non-Hodgkin's lymphoma, the prognostic impact of IPI has been controversial in ENKTL, for most patients diagnosed with ENKTL were in the low-risk IPI group [5]. Ergo, clinical challenges still exist in treatment selection due to the inability to predict the heterogeneous clinical behaviors of stage IE/IIE ENKTL.

Efforts have been made to find out new prognostic indicators for ENKTL. Recently, it has been demonstrated that proinflammatory chemokines and cytokines in the tumor microenvironment contribute to proliferation and survival of malignant cells, angiogenesis, metastasis, subversion of adaptive immunity, reduced response to chemotherapeutic agents, thus affecting survival[6]. Markers of inflammation such as lymphocytes[7], monocytes[8], C-reactive proteins (CRP)[9] had been previously studied in ENKTL patients and were found to have a prognostic value.

Lymphocyte-to-monocyte ratio (LMR), as a converted inflammatory response related biomarker, has been demonstrated to have potential prognostic significance in patients with various types of malignancies[10-14]. However, none of the previous studies looked into the effects of LMR on ENKTL patients. The purpose of this study was to assess the value of the LMR as a predictor of survival in early stage ENKTL patients.

\section{Materials and Methods}

\section{Patient Selection}

This was a retrospective study of an unselected cohort of all newly diagnosed patients with histologically proven ENKTL who were treated at Sun Yat-sen University Cancer Center (Guangzhou, China) between January 1996 and April 2012. The study was performed following approval by the ethic committee of Sun Yat-Sen University Cancer Center. All patients provided written informed consent for the collection and publication of their medical information at the first visit to our center, which was filed in their medical records, and the ethics committees approved this consent procedure. The inclusion criteria were as follows: (1) pathologically confirmed diagnosis of upper aerodigestive tract ENKTL, based on the WHO Classification of Tumours of Hematopoietic and Lymphoid Tissues[1]. (2) Ann Arbor stage IE/IIE. (3) Previously untreated patients. (4) No previous or concomitant malignancies. (5) A complete set of clinical information and follow-up data. (6) Patients with blastic NK-cell lymphoma/ leukemia, aggressive NK-cell lymphoma/leukemia, or peripheral T-cell lymphoma, unspecified, were excluded. (7) Patients with any clinical evidence of acute infection or chronic active inflammatory disease, such as rheumatoid arthritis and virus hepatitis, were also excluded. All pathologic specimens were reviewed and reclassified by central review according to the $\mathrm{WHO}$ criteria for pathologic diagnosis. Antibodies to the following antigens were used for immunophenotype analysis: CD3, CD56, TIA-1, Granzyme-B, CD45RO, CD20, CD79a, CD30, Ki67 and the anaplastic large cell lymphoma kinase. In situ hybridization was used for the detection of EBV-encoded RNA.

\section{Data collection}

The data were collected at diagnosis, including patient demographics, Eastern Cooperative Oncology Group performance status (ECOG PS), primary site, involved sites, systemic B symptoms, complete blood count, serum LDH, Ann Arbor stage, bone marrow status, nuclear magnetic resonance scan of the head and neck and computed tomography scans of the thorax, abdomen and pelvic cavity. All patients were staged using the Ann Arbor staging system. ENKTL was classified into two subsets based on the anatomic distribution of the tumor at presentation [15]. Upper aerodigestive tract NK/T-cell lymphoma was defined as a primary tumor involving the nasal cavity, nasopharynx and upper aerodigestive tract. Only these patients were included in analysis. Local tumor invasiveness was defined as bony invasion or destruction or tumor invasion of the skin or paranasal involvement. Absolute lymphocyte count and absolute monocyte count in peripheral blood were derived from the standard automated complete blood counts which were done at diagnosis. LMR was calculated using the equation $\mathrm{LMR}=$ absolute lymphocyte count / absolute monocyte count.

\section{Response criteria}

Tumor response was assessed radiologically after every two cycles of chemotherapy or before and 
after radiotherapy on the basis of standardized response criteria for non-Hodgkin lymphoma[16]. After completion of treatment, patients were followed up every 3 months in the first 2 years, then every 6 months for the next 3 years and annually thereafter.

\section{Statistical analyses}

All statistical analyses were performed using Statistical Package for the Social Sciences program (SPSS Inc. Chicago, IL, version 15.0 for Windows). Progression-free survival (PFS) was defined as the interval between the date of diagnosis and the date of first relapse, progression, death from any cause, or the last date at which patients were censored. Overall survival (OS) was defined from the date of diagnosis until either the time of death from any cause or the last date at which patients were censored. Categorical characteristics were compared using the chi-square test or Fisher's exact test. Continuous biologic variables were dichotomized. Survival functions were estimated by the Kaplan-Meier method and compared by log-rank test. Multivariate analysis was performed according to the Cox proportional hazards model. Significance was set at $\mathrm{P}<0.05$.

\section{Results}

\section{Patient characteristics}

All patients' characteristics were shown in Table 1. In this cohort of 379 patients with ENKTL, 256 patients $(67.5 \%)$ were men and the mean age was 43.0 years (range, 9-80years). More patients were in Ann Arbor stage I (61.4\%) than in stage II. B symptoms were present in 170 patients $(45.0 \%)$, and serum $\mathrm{LDH}$ levels were elevated in 85 patients $(22.8 \%)$. The present of local tumor invasiveness was common $(66.2 \%)$. The median absolute lymphocyte count at diagnosis was $1.5 \times 10^{9} / \mathrm{L}$ (range 0.1-5.3), while the median absolute monocyte count was $0.5 \times 10^{9} / \mathrm{L}$ (range, 0.1-2.2). For most patients, the absolute lymphocyte count $(90.8 \%)$ and absolute monocyte count $(94.2 \%)$ levels were within the normal range at diagnosis.

\section{Identification of the optimal cut-off value for defining LMR}

The differences in the PFS and OS rates obtained when the patients were stratified into high or low LMR groups using various cut-off values were shown in Table 2. The largest difference was obtained using a cut-off of 2.0 for PFS, and this cut-off point also had significant predictive value for OS. Therefore, this was used to define the low and high LMR groups in subsequent analyses.
Table 1. Baseline clinical characteristics of patients by LMR levels at diagnosis.

\begin{tabular}{|c|c|c|c|c|}
\hline Variable & $\begin{array}{l}\text { No. } \\
(\%)\end{array}$ & $\begin{array}{l}\text { Low LMR group } \\
(\mathrm{LMR} \leq 2.0)\end{array}$ & $\begin{array}{l}\text { High LMR group } \\
\text { (LMR>2.0) }\end{array}$ & $\begin{array}{l}P \\
\text { value }\end{array}$ \\
\hline \multicolumn{5}{|l|}{ Age } \\
\hline$\leq 60$ & 321 & 86 & 235 & \multirow[t]{2}{*}{0.505} \\
\hline$>60$ & 58 & 18 & 40 & \\
\hline \multicolumn{5}{|l|}{ Gender } \\
\hline Male & 256 & 77 & 179 & \multirow[t]{2}{*}{0.097} \\
\hline Female & 123 & 27 & 96 & \\
\hline \multicolumn{5}{|l|}{ B symptoms } \\
\hline+ & 170 & 49 & 121 & \multirow[t]{2}{*}{0.553} \\
\hline - & 207 & 54 & 153 & \\
\hline \multicolumn{5}{|l|}{ Ann Arbor stage } \\
\hline Stage I & 233 & 50 & 183 & \multirow[t]{2}{*}{0.001} \\
\hline Stage II & 146 & 54 & 92 & \\
\hline \multicolumn{5}{|l|}{ LDH } \\
\hline Normal & 288 & 75 & 213 & \multirow[t]{2}{*}{0.298} \\
\hline Elevated & 85 & 27 & 58 & \\
\hline \multicolumn{5}{|l|}{$\begin{array}{l}\text { Local tumor } \\
\text { invasiveness }\end{array}$} \\
\hline Yes & 223 & 66 & 257 & \multirow[t]{2}{*}{0.593} \\
\hline No & 114 & 26 & 88 & \\
\hline \multicolumn{5}{|l|}{ Treatment } \\
\hline Chemotherapy & 80 & 37 & 43 & \multirow[t]{2}{*}{0.000} \\
\hline $\begin{array}{l}\text { Radiotherapy/radiother } \\
\text { apy plus chemotherapy }\end{array}$ & 299 & 67 & 232 & \\
\hline \multicolumn{5}{|l|}{$\begin{array}{l}\text { Absolute lymphocyte } \\
\text { count }\end{array}$} \\
\hline$\leq 1.5 \times 10^{9} / \mathrm{L}$ & 194 & 86 & 108 & \multirow[t]{2}{*}{0.000} \\
\hline$>1.5 \times 10^{9} / \mathrm{L}$ & 185 & 18 & 167 & \\
\hline \multicolumn{5}{|l|}{$\begin{array}{l}\text { Absolute monocyte } \\
\text { count }\end{array}$} \\
\hline$\leq 0.5 \times 10^{9} / \mathrm{L}$ & 190 & 21 & 169 & \multirow[t]{2}{*}{0.000} \\
\hline$>0.5 \times 10^{9} / \mathrm{L}$ & 189 & 83 & 106 & \\
\hline
\end{tabular}

Table 2. Differences in the PFS and OS for patients with ENKTL dichotomized using different cut-off values for the pretreatment LMR.

\begin{tabular}{llllll}
\hline & & PFS & \multicolumn{3}{l}{ OS } \\
\hline $\begin{array}{l}\text { Cut-off, } \\
\text { g/L }\end{array}$ & $\begin{array}{l}\text { number of patients, } \\
\text { low/high }\end{array}$ & chi-square & P value & chi-square & P value \\
\hline 1.8 & $69 / 310$ & 14.804 & 0.000 & 17.230 & 0.000 \\
1.9 & $84 / 295$ & 13.064 & 0.000 & 10.768 & 0.001 \\
2.0 & $104 / 275$ & 15.665 & 0.000 & 14.112 & 0.000 \\
2.1 & $109 / 270$ & 15.456 & 0.000 & 13.434 & 0.000 \\
2.2 & $132 / 247$ & 9.852 & 0.002 & 6.982 & 0.008 \\
2.3 & $140 / 239$ & 8.997 & 0.003 & 6.719 & 0.01 \\
2.4 & $152 / 227$ & 7.678 & 0.006 & 6.624 & 0.01 \\
2.5 & $167 / 212$ & 5.890 & 0.015 & 4.853 & 0.028 \\
\hline
\end{tabular}

\section{Association of LMR with the clinical characteristics}

Baseline clinical characteristics of patients with $\mathrm{LMR} \leq 2.0$ at diagnosis were compared with those with LMR>2.0 (Table 1). No significant between-group difference was observed for age, gender, present of B symptoms and local tumor invasiveness, and $\mathrm{LDH}$ levels, though the proportion of patients classified as Ann Arbor stage II was higher in the low LMR group compared to the high LMR group. 


\section{Treatment modalities and response}

The primary treatment modalities were as follows: (1) chemotherapy alone (80 cases) and (2) radiotherapy alone (9 cases) and (3) both chemotherapy and radiotherapy (290 cases). Radiation therapy was given with daily fractions of 1.8-2.0Gy and five fractions per week using a linear accelerator (6-8 MV). The median radiation dose to the primary tumor site and lymph node negative neck area were 55.4Gy and 46Gy, respectively. Chemotherapy was given over a median of four cycles (range, 1-9 cycles). The regimens of chemotherapy in the initial treatment included: CHOP (cyclophosphamide, doxorubicin, vincristine and prednisolone), CHOPL (CHOP and asparaginase), GELOX (gemcitabine, oxaliplatin and asparaginase), $\mathrm{EPOCH}$ (etoposide, vincristine, doxorubicin, cyclophosphamide and prednisone) and several other regimens used at the discretion of the treating physician. In total, $37.2 \%$ patients received the asparaginase-based regimens. More patients received only chemotherapy in the low LMR group.

In the initial treatment modality, complete remission was achieved in 156 patients (41.2\%), partial remission in 141 patients $(37.2 \%)$, stable disease in 51 patients $(13.5 \%)$, and progressive disease in the remaining 31 patients $(8.2 \%)$, resulting in an objective response rate (ORR) of $78.4 \%$. The ORR after the initial treatment in patients with $L M R \leq 2.0$ was $69.8 \%$, significantly lower than that in patients with LMR $>2.0(83.0 \%, \mathrm{p}=0.000)$.

\section{Survival and prognostic factors}

During a median follow-up time of 28.8 months, $240 / 379$ patients $(63.3 \%)$ experienced tumor progression after treatment (figure 1A). By the end of follow-up, 85/379 patients (22.4\%) had died (figure 1B), including $82(96.5 \%)$ who died of ENKTL and 3 $(3.5 \%)$ who died of other causes. The 2-year and 5 -year PFS rates for the whole cohort were $67.9 \%$, and $57.7 \%$, respectively, while the 2-year and 5-year OS rates were $78.5 \%$, and $72.9 \%$, respectively.

Table 3 and 4 showed the results of univariate and multivariate analysis. LMR at diagnosis was significant predictor for both PFS and OS. Demonstrated in figure 2A, patients in the high LMR group had significantly better PFS than those in the low LMR group (5-year PFS rate: $62.7 \%$ vs. $43.9 \%$ ). As shown in figure $2 \mathrm{~B}$, patients in the high LMR group also had significantly better OS than those in the low LMR group (5-year OS rate: $77.7 \%$ vs. $59.1 \%$ ).

In univariate analysis, clinical features such as age, Ann Arbor stage, local tumor invasiveness, treatment modality and regimens of chemotherapy, laboratory features such as the LDH level, absolute lymphocyte count and LMR were all significant predictors for PFS. Prognostic factors for OS were age, Ann Arbor stage, LDH level, local tumor invasiveness, treatment modality, regimens of chemotherapy, absolute lymphocyte count and LMR. In multivariate analysis, LMR retained its predictive power for both PFS and OS. Age, treatment modality and regimens of chemotherapy were also identified as independent predictive factors for both PFS and OS, while local tumor invasiveness was proved to be an independent predictive factor merely for OS.
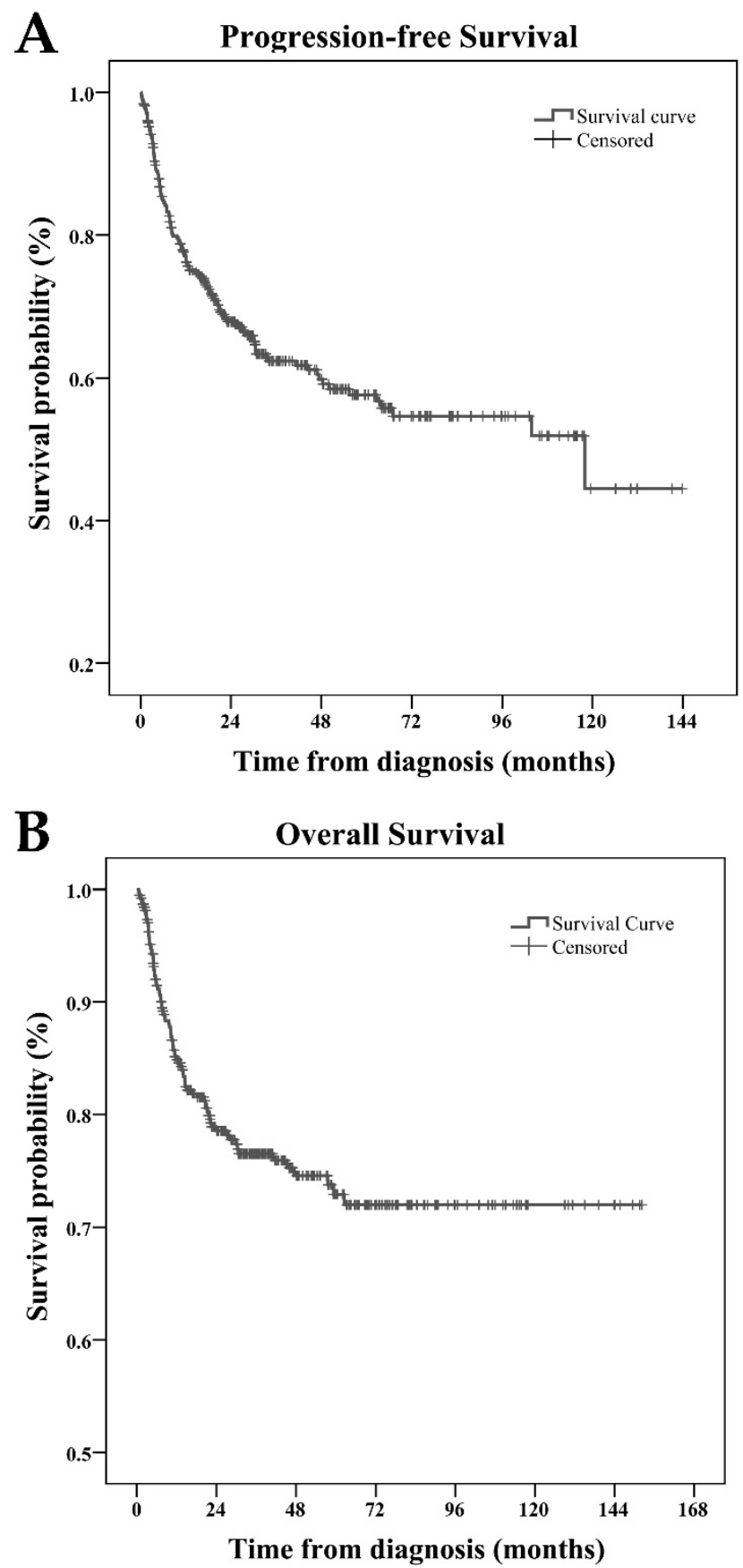

Figure 1. Progression-free survival (A) and overall survival (B) of 379 patients with extranodal natural killer/T-cell lymphoma, nasal type (ENKTL). 
Table 3. Univariate and multivariate analysis of prognostic factors for PFS.

\begin{tabular}{|c|c|c|c|}
\hline \multirow[b]{2}{*}{ Variables } & \multirow{2}{*}{$\begin{array}{l}\text { Univariate } \\
\text { analysis } \\
\text { P value }\end{array}$} & \multicolumn{2}{|c|}{ Multivariate analysis } \\
\hline & & $\mathrm{HR}(95 \% \mathrm{CI})$ & P value \\
\hline $\begin{array}{l}\text { Age } \\
(>60 \text { vs. }<=60)\end{array}$ & 0.004 & $2.031(1.288-3.203)$ & 0.002 \\
\hline $\begin{array}{l}\text { Gender } \\
\text { (male vs. female) }\end{array}$ & 0.586 & $1.158(0.786-1.707)$ & 0.459 \\
\hline $\begin{array}{l}\text { B symptoms } \\
\text { (yes vs. no) }\end{array}$ & 0.803 & $1.303(0.882-1.925)$ & 0.183 \\
\hline $\begin{array}{l}\text { Ann Arbor stage } \\
\text { (II vs. I) }\end{array}$ & 0.032 & $1.354(0.908-2.018)$ & 0.137 \\
\hline $\begin{array}{l}\text { LDH } \\
\text { (elevated vs. normal) }\end{array}$ & 0.030 & $1.246(0.792-1.961)$ & 0.341 \\
\hline $\begin{array}{l}\text { local tumor invasiveness } \\
\text { (yes vs. no) }\end{array}$ & 0.028 & $1.298(0.861-1.956)$ & 0.212 \\
\hline $\begin{array}{l}\text { Treatment } \\
\text { (chemotherapy vs. } \\
\text { radiotherapy or both) }\end{array}$ & 0.000 & $2.746(1.842-4.091)$ & 0.000 \\
\hline $\begin{array}{l}\text { Regimens of chemotherapy } \\
\text { (asparaginase-based vs. } \\
\text { others) }\end{array}$ & 0.000 & $2.367(1.468-3.818)$ & 0.000 \\
\hline $\begin{array}{l}\text { Absolute lymphocyte count } \\
\left(\leq 1.5 \times 10^{9} / \text { L vs. }>1.5 \times 10^{9} / \mathrm{L}\right)\end{array}$ & 0.035 & $1.005(0.667-1.514)$ & 0.981 \\
\hline $\begin{array}{l}\text { Absolute monocyte count } \\
\left(\leq 0.5 \times 10^{9} / \mathrm{L} \text { vs. }>0.5 \times 10^{9} / \mathrm{L}\right)\end{array}$ & 0.152 & $1.045(0.689-1.585)$ & 0.837 \\
\hline $\begin{array}{l}\text { LMR } \\
(\leq 2.0 \text { vs. }>2.0)\end{array}$ & 0.000 & 1.763 (1.119-2.777) & 0.014 \\
\hline
\end{tabular}

Table 4. Univariate and multivariate analysis of prognostic factors for OS.

\begin{tabular}{|c|c|c|c|}
\hline \multirow[b]{2}{*}{ Variables } & \multirow{2}{*}{$\begin{array}{l}\text { Univariate } \\
\text { analysis }\end{array}$} & \multicolumn{2}{|c|}{ Multivariate analysis } \\
\hline & & $\mathrm{HR}(95 \% \mathrm{CI})$ & P value \\
\hline $\begin{array}{l}\text { Age } \\
(>60 \text { vs. }<=60)\end{array}$ & 0.001 & $2.711(1.536-4.787)$ & 0.001 \\
\hline $\begin{array}{l}\text { Gender } \\
\text { (male vs. female) }\end{array}$ & 0.173 & $1.494(0.881-2.534)$ & 0.137 \\
\hline $\begin{array}{l}\text { B symptoms } \\
\text { (yes vs. no) }\end{array}$ & 0.760 & $1.320(0.810-2.151)$ & 0.265 \\
\hline $\begin{array}{l}\text { Ann Arbor stage } \\
\text { (II vs. I) }\end{array}$ & 0.005 & $1.348(0.818-2.222)$ & 0.241 \\
\hline $\begin{array}{l}\text { LDH } \\
\text { (elevated vs. normal) }\end{array}$ & 0.001 & $1.725(0.998-2.982)$ & 0.051 \\
\hline $\begin{array}{l}\text { local tumor invasiveness } \\
\text { (yes vs. no) }\end{array}$ & 0.001 & $2.252(1.246-4.071)$ & 0.007 \\
\hline $\begin{array}{l}\text { Treatment } \\
\text { (chemotherapy vs. } \\
\text { radiotherapy or both) }\end{array}$ & 0.000 & $2.172(1.287-3.667)$ & 0.004 \\
\hline $\begin{array}{l}\text { Regimens of chemotherapy } \\
\text { (asparaginase-based vs. } \\
\text { others) }\end{array}$ & 0.000 & 3.952 (1.984-7.871) & 0.000 \\
\hline $\begin{array}{l}\text { Absolute lymphocyte count } \\
\left(\leq 1.5 \times 10^{9} / \text { L vs. }>1.5 \times 10^{9} / \mathrm{L}\right)\end{array}$ & 0.010 & $0.963(0.567-1.637)$ & 0.890 \\
\hline $\begin{array}{l}\text { Absolute monocyte count } \\
\left(\leq 0.5 \times 10^{9} / \mathrm{L} \text { vs. }>0.5 \times 10^{9} / \mathrm{L}\right)\end{array}$ & 0.925 & $1.631(0.950-2.801)$ & 0.076 \\
\hline $\begin{array}{l}\text { LMR } \\
(\leq 2.0 \text { vs. }>2.0)\end{array}$ & 0.000 & $2.230(1.237-4.018)$ & 0.008 \\
\hline
\end{tabular}

\section{Discussion}

Although ENKTL is a distinctive disease entity with poor prognosis, most of the patients were categorized as low risk based on IPI score, especially for the early stage patients, and standard treatment strategy for this disease has not yet been established.
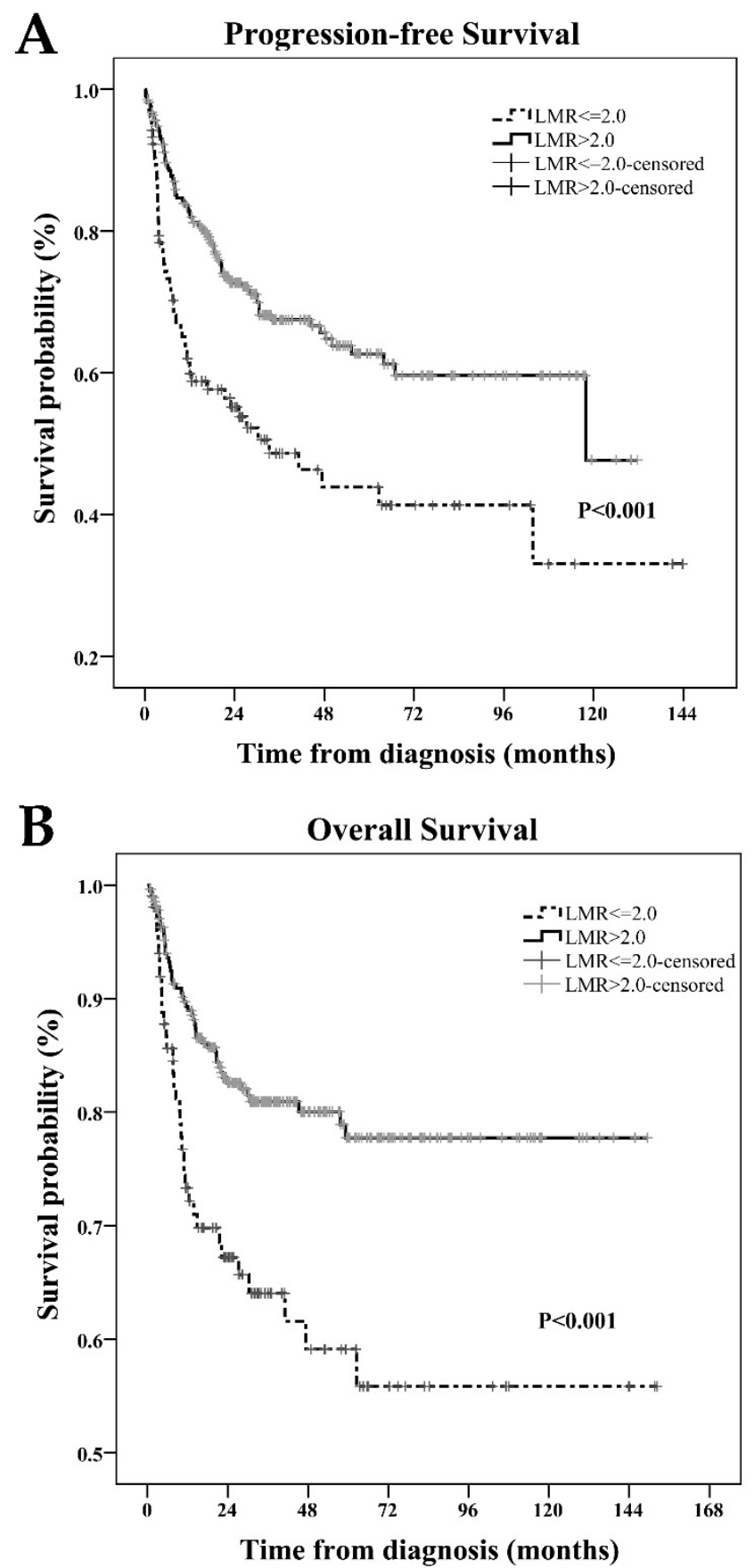

Figure 2. Progression-free survival $(A)$ and overall survival $(B)$ of patients according to lymphocyte/monocyte ratio $(L M R) \leq 2.0$ and $>2.0$ at diagnosis.

For early stage ENKTL, radiation therapy is usually administered. Chemotherapy has been applied in some cancer centers to reduce local relapse and systemic failure [17,18]. However, adding chemotherapy or not remains controversial for early stage ENKTL. Study showed that chemotherapy after radiation therapy did not significantly decrease the systemic failure rate nor improve survival[19]. Meanwhile, there were quite a lot of cases with stage I/II ENKTL who had poor prognosis because of rapid disease progression and recurrence, and intensive 
systemic therapy may be needed for these patients. Therefore, these so called early stage patients need to be subdividing, for predicting the prognosis precisely and guiding the clinical practice appropriately, and multiple new prognostic indicators were proposed for this purpose.

LMR, as an integrated biomarker, has been demonstrated as an independent prognostic indicator in various malignancies[10-14]. However, until now, there has been limited data regarding the role of LMR in the prognosis of ENKTL, and this is the first study to investigate the relationship between LMR and ENKTL to our knowledge. The main finding of this study was that pretreatment LMR is an independent predictor of both OS and PFS in early stage ENKTL patients. Although the low LMR group was associated with advanced Ann Arbor stage and more patients in this group were given only chemotherapy, the prognostic value of LMR remained significant after adjusting for these confounders.

Previous studies reported that asparaginase-based regimens were significantly superior to anthracyline-based regimens for ENKTL in terms of $\mathrm{CR}$ rate and long-term survival outcomes[20]. In this study, we also showed that regimen without asparaginase was an adverse factor for survival. However, after adjusting for this confounder, LMR was still proven to be an independent prognostic factor.

The relationship between chronic inflammation and cancer has been studied for decades. It has been observed that inflammatory diseases increase the risk of developing many types of cancer and that inflammatory cells, chemokines and cytokines are present in the microenvironment of all tumors in experimental animal models and humans from the earliest stages of development[6]. The two pathways connecting inflammation and cancer are elucidated as: the extrinsic pathways, in which inflammatory conditions facilitate cancer development; and the intrinsic pathways driven by oncogenes or tumor suppressor genes that activate the expression of inflammation-related programmers[6]. Previous studies have revealed the link between markers of inflammation such as lymphocytes[7], monocytes[8], platelet lymphocyte ratio[21], CRP[9] and survival in ENKTL patients, and this is synonymous to the association of LMR to ENKTL. There are several potential explanations why chronic inflammation plays such an important role in ENKTL. First, ENKTL is frequently characterized by necrosis and inflammation, and inflammatory cytokines has been shown to be increased in ENKTL[1]. Second, EBV infection is associated with ENKTL. The whole blood EBV DNA load correlates well with treatment response and the increase of EBV DNA load indicates adverse outcome in ENKTL. EBV infection may induce a consistent expression of viral immunogenic proteins, leading to a potent immune response[22]. However, the mechanism by which a systemic inflammatory response might influence survival in ENKTL patients remains unclear.

Inflammation plays an important role in cancer progression and metastasis, which are also influenced by host immunity[23]. Lymphocyte is a key mediator of immunosurveillance, and lymphocyte infiltration into the tumor microenvironment is a prerequisite to an immunologic antitumor reaction[24]. Lymphocytes, including natural killer cells, are important mediators of antibody-dependent cell-mediated cytotoxicity [25], and CD 8+ and CD 4+ T-lymphocytes play an essential role in anti-tumor reaction of the immune system by inducing tumor cell apoptosis[26,27]. Therefore, lymphopenia is considered a surrogate marker of host immunological incompetence. Observation study showed that lymphopenia is an adverse prognostic factor in NHL of various subtypes, including ENKTL [7]. In this study, lymphopenia was associated with impaired PFS and OS in univariate analysis.

Monocyte has also been reported to be associated with adverse prognosis in various malignancies, mostly due to the tumor-associated macrophages (TAMs)[28,29], which are derived from circulating monocyte populations. Mounting evidence showed that TAMs exert activity that is primarily protumoral including promotion of metastasis, immunosuppression, and tumor angiogenesis[29]. Monocyte has also been found to induce the proliferation of natural killer $(\mathrm{NK}) / \mathrm{T}$ lymphoma cells, and NK/T-cell lymphoma cells can produce the IP-10 (a major chemoattractant of human monocytes) which increase monocyte infiltration of the tumor microenvironment[30]. Therefore, there is a positive feedback relationship between monocytes and lymphoma cells in the microenvironment of NK/T-cell lymphoma [30].

In this study, we showed that absolute lymphocyte count was also related to survival outcomes in NKTCL. However, our multivariate analysis showed that only LMR was a prognostic factor for OS and PFS. LMR, as a parameter represented both immunosurveillance and immunosuppression, was shown to outperform the absolute lymphocyte count in the prediction of survival outcome, and this is in line with previous report[10]. Because pre-treatment absolute lymphocyte count and monocyte count are routinely tested in clinical practice, calculation of LMR is easily accessed compared with other parameters. And we 
suggest that LMR should be calculated more often in clinical practice.

However, the appropriate cutoff point for LMR still needs to be investigated. Previous studies have used different cutoff points, ranged from 1.0 to $\geq 4$, and $89 \%$ of these studies have reached a general conclusion that lower LMR group was associated with adverse outcome[31]. Whether there exists a perfect cutoff point for LMR is an important question for its use in clinic and further study may be needed to answer this question.

Other limitations in the current study include: (1) this is a single-institution retrospective study and is therefore limited by the bias inherent in this type of analysis; (2) some patients were treated in the 1990s and received only chemotherapy which may led to inferior survival.

In conclusion, this study demonstrated that LMR at diagnosis is a valuable prognostic indicator of clinical outcome in early stage ENKTL patients. Large prospect study is required to validate this finding, to find out a perfect cutoff point and to provide a better understanding of the mechanisms underlying the association between low LMR level and the adverse clinical outcomes.

\section{Abbreviations}

ENKTL: extranodal natural killer/T-cell lymphoma, nasal type; WHO: World Health Organization; EBV: Epstein-Barr virus; IPI: International Prognostic Index; CRP: C-reactive proteins; LMR: absolute lymphocyte count/absolute monocyte count ratio; LDH: lactate dehydrogenase; PFS: progression-free survival; OS: overall survival; CHOP: cyclophosphamide, doxorubicin, vincristine and prednisolone; CHOPL: CHOP and asparaginase; GELOX: gemcitabine, oxaliplatin and asparaginase; EPOCH: etoposide, vincristine, doxorubicin, cyclophosphamide and prednisone; ORR: objective response rate.

\section{Acknowledgment}

The authors are indebted to all the patients participated in this study at Sun Yat-sen University Cancer Center. They are also grateful to all the pathologists who examined the specimens.

\section{Competing Interests}

The authors have declared that no competing interest exists.

\section{References}

1. Swerdlow S, Campo E, Harris N, et al. WHO Classification of Tumours of Haematopoietic and Lymphoid Tissues, Fourth ed. Colorado, USA: WHO Press; 2008.
2. Li $\mathrm{Y}$, Liu $\mathrm{Q}$, Fang $\mathrm{H}$, et al. Variable clinical presentations of nasal and Waldeyer ring natural killer/T-cell lymphoma. Clin Cancer Res. 2009; 15: 2905-12.

3. Kim TM, Park YH, Lee SY, et al. Local tumor invasiveness is more predictive of survival than International Prognostic Index in stage I(E)/II(E) extranodal NK/T-cell lymphoma, nasal type. Blood. 2005; 106: 3785-90.

4. [No authors listed]. A predictive model for aggressive non-Hodgkin's lymphoma. The International Non-Hodgkin's Lymphoma Prognostic Factors Project. N Engl J Med. 1993; 329: 987-94.

5. Wang L, Chi PD, Chen $\mathrm{H}$, et al. Low level of high-density lipoprotein cholesterol correlates with poor prognosis in extranodal natural killer/T cell lymphoma. Tumour Biol. 2014; 35: 2141-9.

6. Colotta F, Allavena P, Sica A, et al. Cancer-related inflammation, the seventh hallmark of cancer: links to genetic instability. Carcinogenesis. 2009; 30: 1073-81.

7. Huang JJ, Jiang WQ, Lin TY, et al. Absolute lymphocyte count is a novel prognostic indicator in extranodal natural killer/T-cell lymphoma, nasal type. Ann Oncol. 2011; 22: 149-55.

8. Huang J, Li Y, Xia Y, et al. Prognostic significance of peripheral monocyte count in patients with extranodal natural killer/T-cell lymphoma. BMC Cancer. 2013; 13: 222.

9. Li YJ, Li ZM, Xia Y, et al. Serum C-reactive protein (CRP) as a simple and independent prognostic factor in extranodal natural killer/T-cell lymphoma, nasal type. PLoS One. 2013; 8: e64158.

10. Eo WK, Chang HJ, Kwon SH, et al. The Lymphocyte-Monocyte Ratio Predicts Patient Survival and Aggressiveness of Ovarian Cancer. J Cancer. 2016; 7: 289-96.

11. Eo WK, Kwon S, Koh SB, et al. The Lymphocyte-Monocyte Ratio Predicts Patient Survival and Aggressiveness of Endometrial Cancer. J Cancer. 2016; 7: 538-45.

12. Ji $\mathrm{H}$, Xuan $\mathrm{Q}$, Yan $\mathrm{C}$, et al. The prognostic and predictive value of the lymphocyte to monocyte ratio in luminal-type breast cancer patients treated with CEF chemotherapy. Oncotarget. 2016; 23: 34881-9.

13. Li Y, Gu K, Pan Y, et al. Peripheral blood lymphocyte/monocyte ratio at the time of first relapse predicts outcome for patients with relapsed or primary refractory diffuse large B-cell lymphoma. BMC Cancer. 2014; 14: 341.

14. Chan JC, Chan DL, Diakos CI, et al. The Lymphocyte-to-Monocyte Ratio is a Superior Predictor of Overall Survival in Comparison to Established Biomarkers of Resectable Colorectal Cancer. Ann Surg. 2016; [Epub ahead of print].

15. Lee J, Suh C, Park YH, et al. Extranodal natural killer T-cell lymphoma, nasal-type: a prognostic model from a retrospective multicenter study. J Clin Oncol. 2006; 24: 612-8.

16. Cheson B, Horning S, Coiffier B, et al. Report of an international workshop to standardize response criteria for non-Hodgkin's lymphomas. NCI Sponsored International Working Group. J Clin Oncol. 1999; 17: 1244.

17. Guo Y, Lu JJ, Ma X, et al. Combined chemoradiation for the management of nasal natural killer (NK)/T-cell lymphoma: elucidating the significance of systemic chemotherapy. Oral Oncol. 2008; 44: 23-30.

18. Kim W, Song S, Ahn Y, et al. CHOP followed by involved field radiation: is it optimal for localized nasal natural killer/T-cell lymphoma? Ann Oncol. 2001; 12: 349-52

19. Li YX, Liu QF, Wang WH, et al. Failure patterns and clinical implications in early stage nasal natural killer/T-cell lymphoma treated with primary radiotherapy. Cancer. 2011; 117: 5203-11.

20. Wang L, Xia ZJ, Lu Y, et al. A modified international prognostic index including pretreatment hemoglobin level for early stage extranodal natural killer/T cell lymphoma. Leuk Lymphoma. 2015; 56: 3038-44.

21. Wang KF, Chang BY, Chen $X Q$, et al. A prognostic model based on pretreatment platelet lymphocyte ratio for stage IE/IIE upper aerodigestive tract extranodal NK/T cell lymphoma, nasal type. Med Oncol. 2014; 31: 318.

22. Kim HS, Kim KH, Kim KH, et al. Whole blood Epstein-Barr virus DNA load as a diagnostic and prognostic surrogate: extranodal natural killer/T-cell lymphoma. Leuk Lymphoma. 2009; 50: 757-63.

23. Diakos CI, Charles KA, McMillan DC, et al. Cancer-related inflammation and treatment effectiveness. The Lancet Oncology. 2014; 15: e493-503.

24. Dunn G, Old L, Schreiber RD. The immunobiology of cancer immunosurveillance and immunoediting. Immunity. 2004; 21: 137-48.

25. Lin $\mathrm{Y}$, Gustafson $\mathrm{M}$, Bulur $\mathrm{P}$, et al. Immunosuppressive CD14+HLA-DR(low)/- monocytes in B-cell non-Hodgkin lymphoma. Blood. 2011; 117: 872-81.

26. Zikos T, Donnenberg A, Landreneau R, et al. Lung T-cell subset composition at the time of surgical resection is a prognostic indicator in non-small cell lung cancer. Cancer Immunol Immunother. 2011; 60: 819-27.

27. Rosenberg SA. Progress in human tumour immunology and immunotherapy. Nature 2001: 411: 380-4

28. Koh YW, Kang HJ, Park C, et al. The ratio of the absolute lymphocyte count to the absolute monocyte count is associated with prognosis in Hodgkin's lymphoma: correlation with tumor-associated macrophages. Oncologist. 2012; 17: 871-80

29. Galdiero M, Bonavita E, Barajon I, et al. Tumor associated macrophages and neutrophils in cancer. Immunobiology. 2013; 218: 1402-10.

30. Ishii $\mathrm{H}$, Takahara $\mathrm{M}$, Nagato $\mathrm{T}$, et al. Monocytes enhance cell proliferation and LMP1 expression of nasal natural killer/T-cell lymphoma cells by cell 
contact-dependent interaction through membrane-bound IL-15. Int J Cancer. 2012; 130: 48-58.

31. Gu L, Li H, Chen L, et al. Prognostic role of lymphocyte to monocyte ratio for patients with cancer: evidence from a systematic review and meta-analysis. Oncotarget. 2016; 7: 31926-42. 